\title{
Assessment of pediatric cricopharyngeal achalasia with high resolution manometry
}

\author{
Özlem Boybeyi Türer ${ }^{1}$, Numan Demir², Thomas Ciecieraga³ ${ }^{3}$ Rıza Önder Günaydın, \\ Tutku Soyer ${ }^{1}$ \\ Departments of ${ }^{1}$ Pediatric Surgery and ${ }^{4}$ Otorhinolaryngology, Hacettepe University Faculty of Medicine; ${ }^{2}$ Department \\ Physiotherapy and Rehabilitation, Hacettepe University Faculty of Health Sciences; ${ }^{3}$ New York Presbyterian - Weill Cornell \\ Medical College, Department of Pediatric Gastroenterology, New York, USA. E-mail: ozlemboy80@yahoo.com \\ Received: 1st August 2018, Revised: 10th September 2018, Accepted: 17th September 2018
}

SUMMARY: Boybeyi Türer Ö, Demir N, Ciecieraga T, Günaydın RÖ, Soyer T. Assessment of pediatric cricopharyngeal achalasia with high resolution manometry. Turk J Pediatr 2019; 61: 804-809.

Cricopharyngeal achalasia (CPA) is an uncommon cause of oropharyngeal dysphagia (OPD) which is the failure of upper esophageal sphincter (UES) to relax during bolus passage. The diagnostic challenges in OPD have been overcome with the use of high resolution manometry (HRM) in children where a catheter based biomechanical evaluation testing of the oropharyngeal swallowing is performed. Herein, we present a case with severe dysphagia diagnosed as CPA utilizing HRM testing. An 8-year-old boy was seen in our clinic with a two-year history of difficult swallowing, recurrent respiratory tract infections, hypoxia and seizure secondary to aspiration. Esophagography revealed an indentation of the cricopharangeal muscle (CPM) in the cervical part of the esophagus. Videofluroscopic swallow studies (VFSS) revealed cricopharyngeal bar at level of C5-6 and diffuse dysmotility in esophagus. Conventional esophageal manometry revealed absence of peristaltic activity throughout the esophagus. Esophagogastroduodenoscopy revealed narrowing in upper esophagus that with applied force allowed passage of the endoscope. The patient underwent UES dilatation 6 times. He had temporary relief of symptoms. Since he did not have sustained response to dilatation, a botulinium toxin (5IU/each quadrant) injection (BTI) to CPM was performed twice. His symptoms recurred at the end of 3 months. HRM was performed revealing weakness of CPM and uncoordinated contractions and relaxations in UES. We could not reliably differentiate if HRM findings were a sign of primary illness or secondary to BTI. Swallowing rehabilitation was adjusted based on HRM findings. He is still under follow-up with mild dysphagia to certain solids. CPA is an uncommon cause of dysphagia with limited therapeutic options. HRM should be performed in OPD before considering any treatment modality including esophageal dilatation and BTI. HRM has become gold standard diagnostic tool in OPD that provides objective evaluation of pharyngeal and UES motility in children.

Key words: cricopharyngeal achalasia, dysphagia, high resolution manometry.

Cricopharyngeal achalasia (CPA) is an uncommon cause of oropharyngeal dysphagia (OPD) especially in children and it was first described in children by Utian and Thomas in 1967. ${ }^{1,2}$ CPA is caused by failure of the upper esophageal sphincter (UES) to relax for a bolus during swallowing. ${ }^{1,3}$ The cricopharyngeal muscle (CPM) which is the main component of UES is tonically contracted at rest and relaxes during normal swallowing to enable airway protection and propulsion of food bolus., ${ }^{4}$ The failure of relaxation leads to inability to swallow and increases risk for aspiration of residual food in pharynx. ${ }^{4}$ 
The clinical presentation of CPA includes chocking, coughing, dysphagia, poor weight gain and respiratory problems secondary to aspiration. Videofluroscopic swallow studies (VFSS), esophagoscopy and manometry are the main tools used in diagnosis. ${ }^{3,4}$ Although esophageal manometry is the gold standard for diagnosis of esophageal motility disorders, interpretation of UES functionality is challenging due to its anatomical and functional complexity, especially in pediatric population. The diagnostic challenges in OPD have been overcome with the use of high resolution manometry (HRM) in children which is a catheter based neuromuscular evaluation test of oropharyngeal swallowing. ${ }^{6}$ HRM with esophageal pressure topography improved the diagnostic accuracy and characterization of esophageal motility disorders in children. ${ }^{4,6}$ The treatment options in CPA include mechanical dilatation of UES, botulinum toxin injection (BTI) to CPM and open or endoscopic cricopharyngeal myotomy. ${ }^{3}$

Herein, we present a case of an 8-year-old boy with severe dysphagia diagnosed with CPA and managed with mechanical dilatation and BTI. Since no sustained improvement was observed, he underwent HRM testing.

\section{Case Report}

The patient's anonymity is protected and informed consent has been taken. An 8-yearold boy was seen in our clinic with a two-year history of difficulty in swallowing solid foods, recurrent respiratory infections, hypoxia and seizure secondary to aspiration. He was born at full-term gestation, without any problems. He had no congenital abnormalities. His family history was unremarkable. He had no feeding problem during infancy. He was healthy before age 6 , but he was having recurrent respiratory tract infections since early infancy. At age 6 , he developed dysphagia to solids. He was evaluated at a regional hospital and esophagography was performed revealing indentation of CPM in cervical part of esophagus, slightly narrowing the lumen. Esophageal mechanical dilatation was performed however his swallowing did not improve. We then saw him for a second opinion.
On admission, he was unable to swallow solid foods and had coughing episodes. He was aspirating even his saliva and needed to be hospitalized because of respiratory failure and seizure secondary to aspiration 2 times in the last year. His weight was $19 \mathrm{~kg}(10 \mathrm{p})$ and height was $119 \mathrm{~cm}(50 \mathrm{p})$ revealing mild malnutrition. We performed an esophagogastrography revealing CPM indentation and dilatation of esophagus proximal to the indentation of CPM with irregular contractions in mid- and distal esophagus without gastroesophageal reflux noted (Fig. 1). Videofluroscopic swallow studies (VFSS) revealed cricopharyngeal bar at level of C5-6, aspiration with liquids and diffuse dysmotility in esophagus. Conventional esophageal manometry showed hypopersistalsis in the entire esophagus, however, the UES could not be reliably evaluated (Fig. 2). His complaints did not resolve with swallowing rehabilitation or use of thickeners. Neurological evaluation together with brain magnetic resonance imaging (MRI) and electroencephalogram (EEG) were performed revealing normal findings except for mild pontocerebellar atrophy, presumably secondary to hypoxia attacks. The patient was evaluated in our Swallowing Research Center to define an underlying disease but no other systemic disease was found responsible for the motility problem.

Esophagoscopy showed narrowing in an upper esophagus that allows passage of the endoscope confirming diagnosis of CPA. Patient underwent mechanical esophageal dilatation with boogie 6 times (45 and $48 \mathrm{~F}$ ). He had temporary relief of symptoms, but had dysphagia during follow-up. Botulinium toxin (5IU/each quadrant, Botox, Allergan Med., Istanbul, Turkey) injection to CPM was performed two times, but dysphagia has recurred. Then, a HRM was performed 8 weeks after the last BTI revealing weakness of CPM and uncoordinated contractions and relaxations in UES (Fig. 3). We could not differentiate whether this topography was a sign of primary illness or secondary to BTI. Therefore, another HRM study has been planned 6 months after the BTI injection. Swallowing rehabilitation was modified based on HRM findings. The therapy program consisted of thermal 

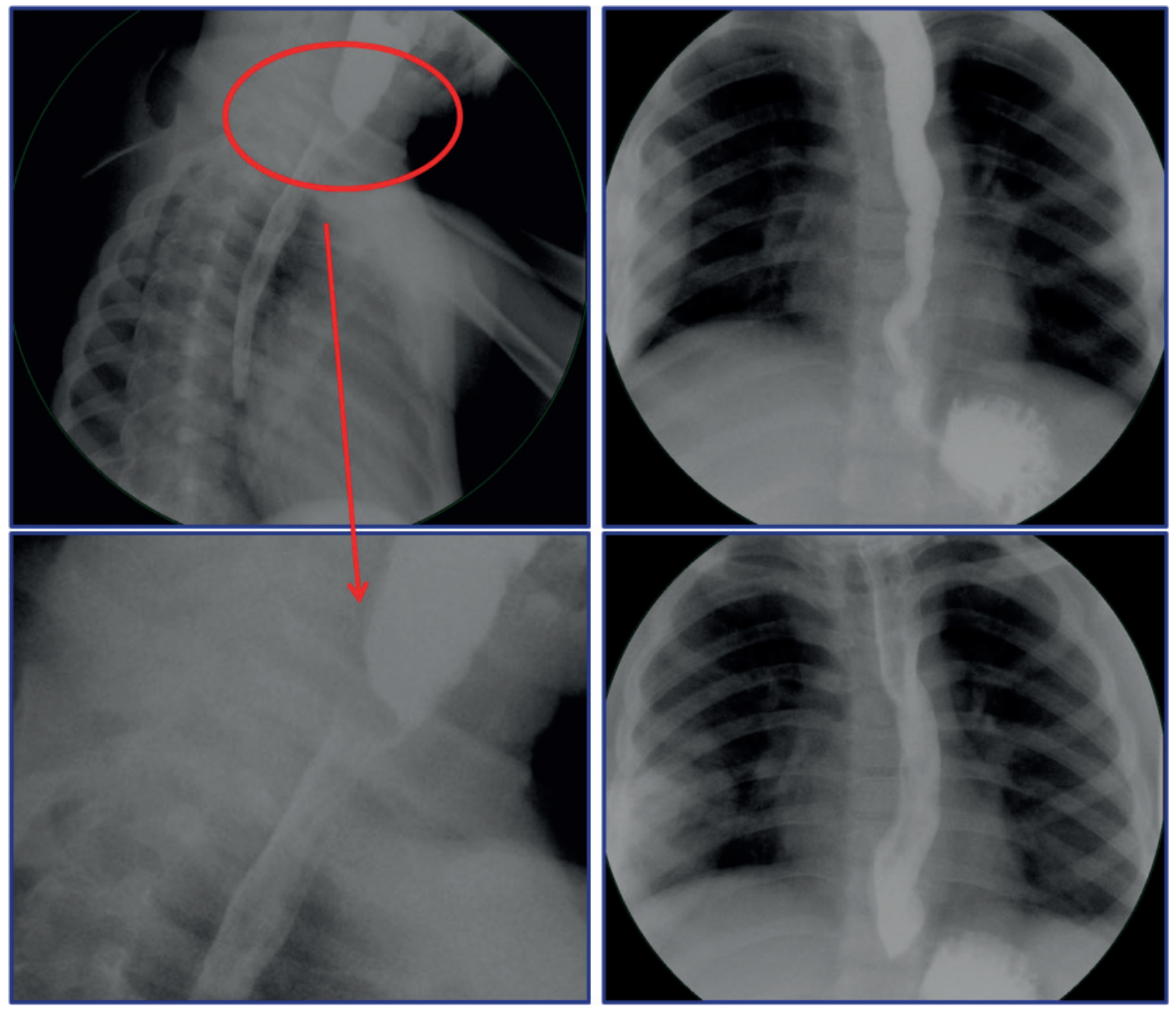

Fig. 1. The esophagogastrography revealing the indentation of cricopharangeal muscle in cervical part of esophagus (arrow), dilatation in esophagus proximal to the indentation, and irregular contractions in midand distal esophagus.

tactile stimulation to trigger swallowing reflex, laryngeal mobilization to support hypolaryngeal elevation, shaker exercise to improve pharyngeal contractions, effortful swallow to increase posterior tongue base movement to facilitate bolus clearance. It was suggested that the exercises be performed twice a day for 3 days a week. He is still under follow-up with mild dysphagia with certain solid foods.

\section{Discussion}

The UES is a high-pressure zone between the pharynx and cervical esophagus. ${ }^{7}$ UES prevents air from entering the esophagus during inspiration and esophageal contents from being aspirated into airways. ${ }^{7}$ The UES pressure is mainly provided by CPM which is contracted in its tonic state and relaxes transiently during swallowing, belching and vomiting. ${ }^{7}$ The failure of relaxation of CPM leads to difficulty in swallowing namely oropharyngeal dysphagia., ${ }^{4,7}$

Cricopharyngeal achalasia (CPA) is an uncommon cause of OPD especially in children. ${ }^{1,3}$ The clinical presentation of CPA includes chocking, coughing, dysphagia, weight loss and respiratory problems secondary to aspiration. The diagnosis is usually made with VFSS which shows the indentation of CPM specifically an indication of CPA. ${ }^{3}$ 


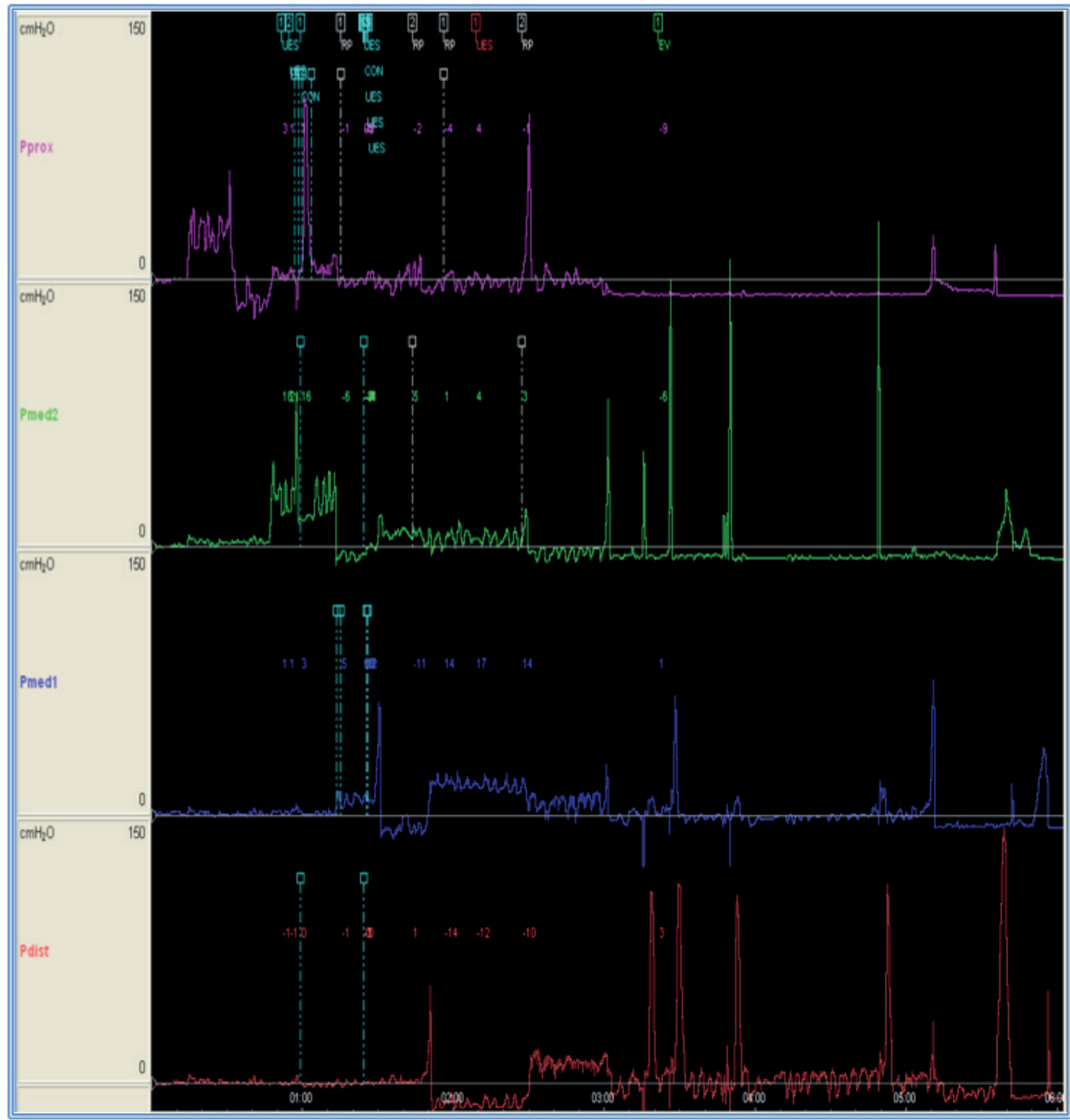

Fig. 2. The conventional esophageal manometry revealing hypopersistalsis in the entire esophagus.

The high resolution esophageal manometry is the gold standard for diagnosis of CPA; however it is hard to interpret the manometric examination of UES because of its complex structure and variations in function. ${ }^{4,6}$ The use of HRM in children has increased the accuracy of diagnosis and improved characterization of esophageal motility disorders in children., 4,6 The indentation of CPM in esophagography, cricopharyngeal bar at level of C5-6 and aspiration with liquids in VFSS made us to diagnose the present case as CPA. However, the conventional manometry was unable to show the increased pressure in UES although it revealed diffuse absence of peristaltic activity in esophageal body.

The treatment options of CPA include mechanical dilatation of UES, BTI to CPM and open orendoscopic cricopharyngeal myotomy. ${ }^{3,9}$ We performed esophageal dilatation several times, however, only temporary relief was obtained. Therefore, we decided to inject botulinum toxin to CPM. BTI is reported to be beneficial in preventing aspiration and relief 

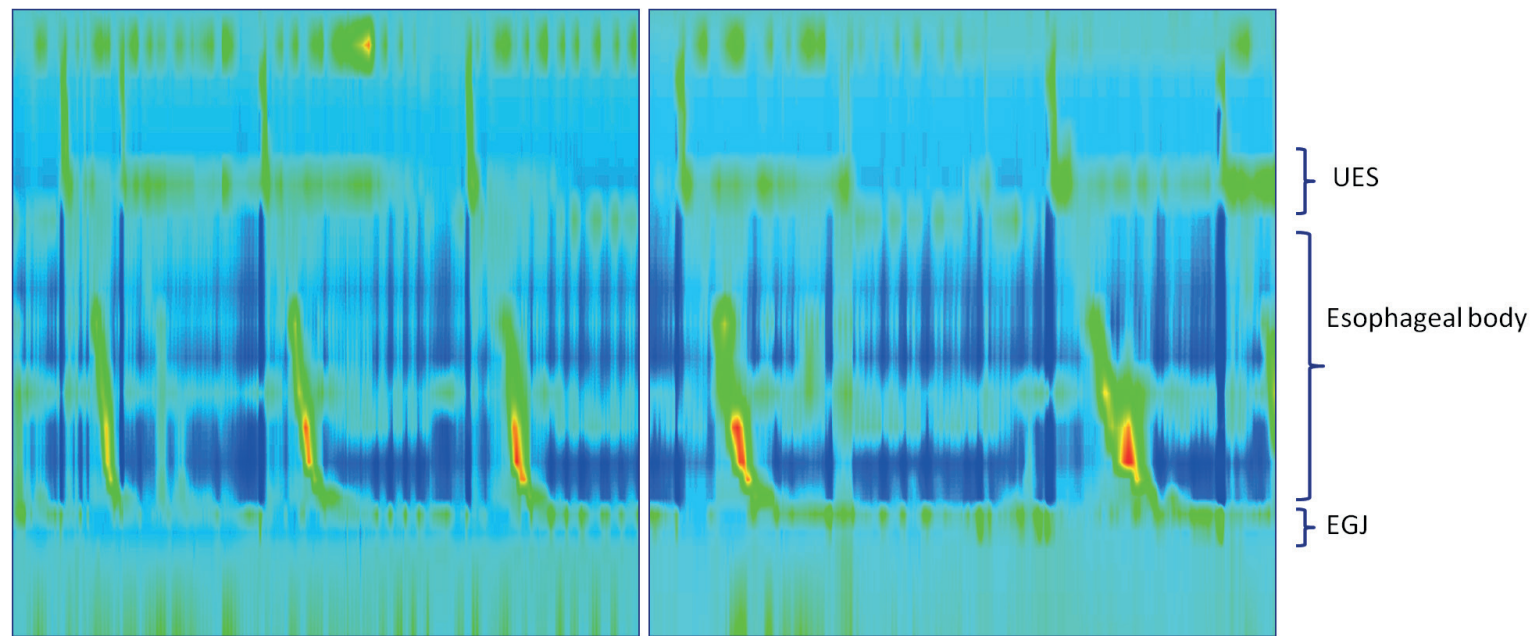

Fig. 3. The high-resolution manometry revealing weakness of CPM and uncoordinated contractions and relaxations in UES (UES: upper esophageal sphincter, EGJ: esophagogastric junction).

of dysphagia. ${ }^{1,10}$ The possible complications are leakage of botulinum toxin to adjacent tissues, improper injection affecting vocal cords which can worsen swallowing. ${ }^{10}$ The patient had relief of symptoms after BTI but recurrence was seen 2 months later. Although there are several reports revealing beneficial effects of dilatation and BTI, we could not achieve such a success in the present case. ${ }^{1,10-}$ ${ }_{13}$ A high resolution esophageal manometry revealed weakness of CPM and uncoordinated contractions and relaxations of UES. Unresponsiveness of our case to dilatation and BTI could represent presence of an additional motility disorder without any underlying systemic disease. Since the effect of BTI is temporary, 'wait and watch' is planned for the patient. However, we should reconsider our swallowing therapy if the HRM findings of UES were primary. Therefore, we decided to modify the swallowing rehabilitation and follow the patient. He is still under follow-up with mild improvement.

In conclusion, CPA is an uncommon cause of dysphagia with limited therapeutic options. HRM should be performed in CPD before considering any treatment modality including esophageal dilatation and BTI. HRM has become gold standard diagnostic tool in CPD that provides objective evaluation of pharyngeal and UES motility in children.

\section{REFERENCES}

1. Scholes MA, McEvoy T, Mousa H, Wiet GJ. Cricopharyngeal achalasia in children: botulinum toxin injection as a toll for diagnosis and treatment. Laryngoscope 2014; 124: 1475-1480.

2. Utian HL, Thomas RG. Cricopharyngeal incoordination in infancy. Pediatrics 1969; 43: 402406.

3. Huoh KC, Messner AH. Cricopharyngeal achalasia in children: indications for treatment and management options. Curr Opin Otolaryngol Head Neck Surg 2013; 21: 576-580.

4. Ferris L, Rommel N, Doeltgen S, et al. Pressure-flow analysis for the assessment of pediatric oropharyngeal dysphagia. J Pediatr 2016; 177: 279-285.e1.

5. Mohan S, Bowe SN, Hirner LM, Zar-Kessler C, Hartnick CJ. Modified approach for pediatric external cricopharyngeal myotomy. Int J Pediatr Otorhinolaryngol 2018; 105: 111-114.

6. Bhatia SJ, Shah C. How to perform and interpret upper esophageal sphincter manometry. J Neurogastroenterol Motil 2013; 19: 99-103.

7. Sivarao DV, Goyal RK. Functional anatomy and physiology of the upper esophageal sphincter. Am J Med 2000; 108(Suppl 4a): 27S-37S.

8. Edeani F, Malik A, Kaul A. Characterization of esophageal motility disorders in children presenting with dysphagia using high-resolution manometry. Curr Gastroenterol Rep 2017; 19: 13.

9. Chun R, Sitton M, Tipnis NA, et al. Endoscopic cricopharyngeal myotomy for management of cricopharyngeal achalasia in an 18-month-old child. Laryngoscope 2013; 123: 797-800. 
10. Barnes MA, Ho AS, Malhotra PS, Koltai P, Messner A. The use of botulinum toxin for pediatric cricopharyngeal achalasia. Int $\mathrm{J}$ Pediatr Otorhinolaryngol 2011: 75: 1210-1214.

11. Gollu G, Demir N, Ates U, et al. Effective management of cricopharyngeal achalasia in infants and children with dilatation alone. J Pediatr Surg 2016; 51: 17511754 .
12. Erdeve O, Kologlu M, Saygili B, Atasay B, Arsan S. Primary cricopharyngeal achalasia in a newborn treated by balloon dilatation: a case report and review of the literature. Int J Pediatr Otorhinolaryngol 2007; 71: 165-168.

13. Akcaboy M, Zorlu P, Demir N, Acoglu EA, Oguz MM, Senel S. A rare cause of dysphagia in children: primary cricopharyngeal achalasia. J Pediatr Child Health 2017; 53: 827-828. 